\title{
On the Largest Prime Divisor of an Odd Perfect Number
}

\author{
By Peter Hagis, Jr. and Wayne L. McDaniel
}

Abstract. It is shown here that if $n$ is odd and perfect, then $n$ has a prime divisor which exceeds 11200.

1. Introduction. If $\sigma(n)$ denotes the sum of the positive divisors of the natural number $n$, then $n$ is said to be perfect if

$$
\sigma(n)=2 n .
$$

Twenty-four even perfect numbers are known at present, but whether or not an odd perfect number exists is an open question. However, many necessary conditions for the existence of an odd perfect number are known. The oldest of these is due to Euler who proved that if $n$ is odd and perfect, then

$$
n=p_{0}^{\alpha_{0}} p_{1}^{\alpha_{1}} \cdots p_{t}^{\alpha_{t}}
$$

where the $p_{i}$ are distinct primes, $p_{0} \equiv \alpha_{0} \equiv 1(\bmod 4)$, and $\alpha_{i}$ is even for $i>0$. In 1944 Kanold [2] showed that at least one of the $p_{i}$ is greater than or equal to 61. Our purpose here is to show that Kanold's result can be significantly improved. Thus, we shall prove the following result.

THEOREM. If $n$ is an odd perfect number, then $n$ is divisible by a prime which exceeds 11200.

Our proof is computational in nature in as much as it is based on a search for the prime factors less than 11200 of $\sigma\left(p^{\alpha}\right)$ for a large number of values of $p$ and $\alpha$. This search was carried out using the CDC 6400 at the Temple University Computing Center and utilized the fact that $\sigma\left(p^{\alpha}\right)=\prod F_{d}(p)$ where $F_{d}$ is the $d$ th cyclotomic polynomial and $d$ runs over the divisors of $\alpha+1$ which exceed 1 . The theorems concerning the prime divisors of cyclotomic polynomials which may be found in Section 48 of [3] facilitate the search.

2. Some Preliminaries. According to a theorem of Kanold [1], if $P$ is the greatest of the $p_{i}$ in (2) and $\beta$ is the maximum of the $\alpha_{i}$, then $P>2(\beta+1)$. It follows that for our purpose we may assume that each $\alpha_{i}$ is less than 5599. Thus, in the remainder of this paper, $n$ will always denote an odd perfect number whose canonical factorization is given by (2), with every $\alpha_{i}<5599$.

If $p^{\alpha} \mid n$ but $p^{\alpha+1} \nmid n$, we shall write $p^{\alpha} \| n$. In this case, we note from (1) and the fact that the $\sigma$-function is multiplicative that every odd prime which divides $\sigma\left(p^{\alpha}\right)$ also divides $n$.

3. Phase I. Suppose that $p$ is a prime and $\alpha$ is a natural number. We shall say that $p^{\alpha}$ is $(5599 ; 11200)$-acceptable or simply acceptable if $\alpha<5599$ and every prime

Received July 31, 1972.

AMS (MOS) subject classifications (1970). Primary 10A20. 
which divides $\sigma\left(p^{\alpha}\right)$ is less than 11200. (We consider only even values of $\alpha$ unless $p=4 k+1$ in which case we also consider those $\alpha$ of the form $4 k+1$.) For example, since $\sigma\left(31^{\alpha}\right)$ is divisible by a prime greater than 11200 for $\alpha=4,6,8, \cdots, 5598$ the only acceptable power of 31 is $31^{2}$. Clearly, our theorem is true if at least one of the $p_{i}^{\alpha_{i}}$ in (2) is unacceptable. Assuming that each prime power in (2) is acceptable we shall now show that $n$ is not divisible by certain "small" primes.

(A) $31 \nmid n$. For the only acceptable power of 31 is $31^{2}$. Since $\sigma\left(31^{2}\right)=3 \cdot 331$, we see that if $31 \mid n$ then also $331 \mid n$. Only $331^{2}$ is acceptable. But $5233 \mid \sigma\left(331^{2}\right)$, and only 5233 is acceptable. $2617 \mid \sigma(5233)$ and only 2617 is acceptable. We conclude that 5233||$n$ and 2617||$n$ which contradicts (2).

(B) $67 \nmid n$. For only $67^{2}$ is acceptable and $\sigma\left(67^{2}\right)=3 \cdot 7^{2} \cdot 31$. Therefore, if $67 \mid n$ then $31 \mid n$. This contradicts (A).

(C) $19 \nmid n$. The only acceptable powers of 19 are $19^{2}$ and $19^{4}$. But $911 \mid \sigma\left(19^{4}\right)$, and no power of 911 is acceptable. $\sigma\left(19^{2}\right)=3 \cdot 127$ and only $127^{2}$ is acceptable. $5419 \mid \sigma\left(127^{2}\right)$ and only $5419^{2}$ is acceptable. Since $31 \mid \sigma\left(5419^{2}\right), 19^{2} \| n$ is impossible.

(D) $11 \nmid n$. Only $11^{2}$ and $11^{4}$ are acceptable. If $11^{2} \| n$ then, since $\sigma\left(11^{2}\right)=7 \cdot 19$, $19 \mid n$ which contradicts (C). If $11^{4}|| n$ then 5.3221 |n. Only 3221 is acceptable and $\sigma(3221)=2 \cdot 3^{2} \cdot 179$. Only $179^{2}$ is acceptable and $7 \mid \sigma\left(179^{2}\right)$. Therefore, $3 \cdot 5 \cdot 7 \mid n$ which is impossible. (See (g) on p. 109 of [1].)

(E) $37 \nmid n$. Only $37,37^{2}, 37^{4}, 37^{5}$ are acceptable. But $19|\sigma(37) ; 67| \sigma\left(37^{2}\right)$; $11\left|\sigma\left(37^{4}\right) ; 19\right| \sigma\left(37^{5}\right)$.

(F) If $29 \mid n$, then 29||$n$. For the only acceptable powers of 29 are $29,29^{2}, 29^{5}$ and 67 divides both $\sigma\left(29^{2}\right)$ and $\sigma\left(29^{5}\right)$.

(G) $7 \nmid n$. The acceptable powers of 7 are $7^{2}, 7^{4}, 7^{6}, 7^{8}$. Both $\sigma\left(7^{2}\right)$ and $\sigma\left(7^{8}\right)$ are divisible by 19 . Therefore, $7^{2} \nmid n$ and $7^{8} \nmid n$. If $7^{4} \| n$ then $2801 \mid n$. Only 2801 and $2801^{2}$ are acceptable, and $37\left|\sigma\left(2801^{2}\right) .467\right| \sigma(2801)$ and no power of 467 is acceptable. We conclude that $7^{4} \backslash n \cdot \sigma\left(7^{6}\right)=29.4733$, and only 4733 is acceptable. If $7^{6} \| n$, it then follows from (F) that $29 \| n$ and $4733 \| n$. This contradicts (2).

(H) $23 \nmid n, 47 \nmid n, 83 \nmid n$. For each of the primes $23,47,83$, only the second power is acceptable. But $7\left|\sigma\left(23^{2}\right) ; 37\right| \sigma\left(47^{2}\right) ; 19 \mid \sigma\left(83^{2}\right)$.

(I) $11197 \nmid n$. For only 11197 is acceptable and $11 \mid \sigma(11197)$.

We collect these results as a lemma.

LEMMA. If every prime power in the canonical factorization of the odd perfect number $n$ is acceptable, then $n$ is not divisible by any prime in the set $A$ where $A=\{7,11,19$, $23,31,37,47,67,83\}$.

4. Phase II. Let $q$ be the smallest prime which divides $n$, and let $B(q)$ denote the set of odd primes which are less than $q$. If $p \notin A$ we shall say that $p^{\alpha}$ is $(5599 ; 11200$; $A ; q$ )-feasible or feasible with respect to $q$ or simply feasible if $p^{\alpha}$ is acceptable and if also no prime divisor of $\sigma\left(p^{\alpha}\right)$ belongs to the set $A \cup B(q)$. Our theorem is obviously true if $p^{\alpha}$ is not feasible and $p^{\alpha} \| n$.

If $q \geqq 101$ then, according to the table in [4], $p \mid n$ where $p \geqq 11197$. Referring to (I) and our Lemma, we see then that for our purpose we can assume that $q$ is in the set $S=\{3,5,13,17,29,41,43,53,59,61,71,73,79,89,97\}$. A computer search revealed that, unless $q \in T$ where $T=\{3,17,41,59,71,79,89\}$, then $q$ has no feasible powers with respect to $q$. Therefore, our theorem is certainly true unless $q$, the smallest prime dividing $n$, belongs to $T$ and every prime power in the canonical factorization of 
$n$ is feasible with respect to $q$. We shall complete our proof by showing that for each prime in $T$ the assertion that the prime in question is $q$ is incompatible with the requirement of feasibility for each of the $p_{i}^{\alpha_{i}}$ in (2).

(a) $q \neq 3$. The only feasible powers of 3 are $3^{2}, 3^{6}, 3^{8}, 3^{26}$. If $3^{2} \| n$ then $13 \mid n$. The only feasible power of 13 is $13^{2}$, and $\sigma\left(13^{2}\right)=3 \cdot 61$. Only $61^{2}$ is feasible, and $\sigma\left(61^{2}\right)=$ $3 \cdot 13 \cdot 97$. Only $97^{2}$ is feasible, and $\sigma(97)^{2}=3 \cdot 3169$. Therefore, $3^{3} \mid n$ which contradicts $3^{2} \| n . \sigma\left(3^{6}\right)=1093$, and only 1093 is feasible. $547 \mid \sigma(1093)$, and only $547^{2}$ is feasible. Since $163 \mid \sigma\left(547^{2}\right)$ and no power of 163 is feasible, we see that $3^{6} \nmid n .757$ divides both $\sigma\left(3^{8}\right)$ and $\sigma\left(3^{26}\right)$. Only 757 is feasible and $379 \mid \sigma(757)$. Only $379^{2}$ is feasible and $787 \mid \sigma\left(379^{2}\right)$. No power of 787 is feasible. Therefore, $3^{8} \not n$ and $3^{26} \nmid n$.

(b) $q \neq 17.17^{2}$ is the only feasible power of $17 . \sigma\left(17^{2}\right)=307$, and only $307^{4}$ is feasible. But $1051 \mid \sigma\left(307^{4}\right)$, and 1051 has no feasible powers. We conclude that $17^{2} \not n$.

(c) $q \neq 41$. Only $41^{2}$ is feasible, and $\sigma\left(41^{2}\right)=1723.1723$ has no feasible powers.

(d) $q \neq 59.59^{2}$ is the only feasible power of 59. But $\sigma\left(59^{2}\right)=3541$, and 3541 has no feasible powers.

(e) $q \neq 71$. The only feasible power of 71 is $71^{2} . \sigma\left(71^{2}\right)=5113$, and only 5113 is feasible. $2557 \mid \sigma(5113)$ and only 2557 is feasible. Therefore, $5113 \| n$ and $2557 \| n$ which contradicts (2).

(f) $q \neq 79$. Only $79^{6}$ is feasible. But $1289 \mid \sigma\left(79^{6}\right)$, and 1289 has no feasible powers.

(g) $q \neq 89.89^{2}$ and $89^{4}$ are both feasible. But $\sigma\left(89^{2}\right)=8011$ and $131 \mid \sigma\left(89^{4}\right)$; and neither 8011 nor 131 have any feasible powers.

5. Concluding Remark. It seems very likely that the attack of this paper can be used to obtain a much greater lower bound than 11213 for the maximum prime divisor of an odd perfect number. However, both the effort and the computer time required appear to increase at an exponential rate.

Department of Mathematics

Temple University

Philadelphia, Pennsylvania 19122

Department of Mathematics

University of Missouri-St. Louis

St. Louis, Missouri 63121

1. H.-J. KaNOLD, "Untersuchungen über ungerade vollkommene Zahlen," J. Reine Angew. Math., v. 183, 1941, pp. 98-109. MR 3, 268.

2. H.-J. Kanold, "Folgerungen aus dem Vorkommen einer Gauss'schen Primzahl in der Primfaktorenzerlegung einer ungeraden vollkommenen Zahl," J. Reine Angew. Math., v. 186, 1944, pp. 25-29. MR 6, 255.

3. T. Nagell, Introduction to Number Theory, Wiley, New York; Almqvist \& Wiksell, Stockholm, 1951. MR 13, 207.

4. K. Norton, "Remarks on the number of factors of an odd perfect number," Acta Arith., v. 6, 1960, pp. 365-374. MR $26 \# 4950$. 